\title{
Su Lectura Dantis di Carmelo Bene (con un'apparizione di Salvatore Sciarrino)
}

\author{
Simone Caputo \\ Università di Roma "La Sapienza" \\ caputo.simone@gmail.com
}

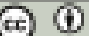

\section{Riassunto}

L'indagine propone una disamina di Lectura Dantis, lavoro di Carmelo Bene del I98I, alla luce delle riflessioni di Vincenzo Caporaletti sul Principio audiotattile, utilizzate in questo caso di studio per un primo avvicinamento al teatro "sonoro" di Carmelo Bene. L'idea di affrontare Lectura Dantis dal versante della musica e non da quello del teatro deriva dalla lettura di due scritti dedicati a Bene: Antropologia di una macchina attoriale di Piergiorgio Giacchè e Un muezzin per Dante di Andrea Cortellessa, che ravvisano nelle qualità soniche dei lavori che Bene produsse tra la fine degli anni Settanta e gli Ottanta, alcuni dei cardini del suo essere "dentro e contro" l'arte della scena. Il principio audiotattile, attraverso la "codifica neoauratica" (la registrazione sonora), invita alla scoperta dei valori che attraversano Lectura Dantis di Carmelo Bene, restituendo una lettura organica di un fenomeno complesso, difficilmente categorizzabile, che nacque dall'intersezione tra una commemorazione pubblica (la celebrazione dell'anniversario della Strage di Bologna, 1980), un canone della letteratura italiana (la Divina Commedia), una performance scenica atipica (Bene si esibì sulla Torre degli Asinelli di Bologna), phoné e musica (composta, montata, amplificata).

Parole chiave: Carmelo Bene; Lectura Dantis; teatro sonoro.

\begin{abstract}
This paper offers an analysis of Lectura Dantis, a work of Carmelo Bene from 198I, in the light of the reflections by Vincenzo Caporaletti and his Principio audiotactile and the use in this case of the study for a first approach to the "hearing" theatre of Carmelo Bene. The idea of facing a Lectura Dantis with music and theatrical effects comes from the reading of the two writings dedicated to Bene: Antropología di una macchina attoriale di Piergiorgio Giacchè and Un muezzin per Dante by Andrea Cortellessa that analyses the sonic quality of the work that Bene produced at the late I970s and early I980s, some of his cornerstones being "in and against" the art scene. The audio tactile principle of his "codifica neoaurica" (the sonic register) invites and reveals the values that are to be found in the Lectura Dantis by Carmelo Bene, replacing an organic reading of a complex phenomenon, of difficult categorization, that is born from the intersection of a public commemoration (the celebration of the massacre of Bologna, 1980), a canon of Italian literature (The Divine Comedy), an atypical performance (Bene performed from the Tower of Bologna), phone and music (composed, produced and amplified).
\end{abstract}

Keywords: Carmelo Bene; Lectura Dantis; Sonic theatre. 


\section{Premessa}

T a presente indagine si propone di tentare una disamina di Lectura Dantis, ${ }^{\mathrm{I}}$ Llavoro di Carmelo Bene del $198 \mathrm{I},{ }^{2}$ alla luce delle riflessioni elaborate da Vincenzo Caporaletti sul Principio audiotattile, quale elemento cardine nel processo di produzione segnica delle musiche non appartenenti alla tradizione eurocolta, e utilizzato in questo caso di studio, producendo un'ovvia forzatura, per un primo avvicinamento al teatro "sonoro" di Carmelo Bene. ${ }^{3}$ Per Principio audiotattile Caporaletti intende quel principio

sintetico, organico, globale di adesione somatico-comportamentistica alla dimensione sonora, in funzione del quale il materiale musicale si configura ed organizza in uno specifico senso linguistico-formale. Dà luogo ad una modulazione fisico-gestuale di energie sonoro-musicali, agendo in modo determinante ai fini della strutturazione del testo musicale (Caporaletti 2005: 106). 4

Tale concezione ha lo scopo di modellizzare fenomeni creativi caratteristici di quelle musiche contemporanee non riconducibili agli svolgimenti della tradizione d'arte scritta occidentale (con riferimento particolare al periodo romantico e post-romantico).

Va ricordato che la teoria mediologica audiotattile trae forza e suggestione primariamente da un'applicazione in campo musicologico di concetti elaborati dalla cosiddetta scuola di Toronto (segnatamente, Marshall Mc Luhan e Derrick De Kerckhove): 5 in questo ambito il ruolo del medium comunicativo/ formativo, viene considerato come non neutrale ai fini della elaborazione e

* Un particolare ringraziamento va a Goffredo Fofi e Piergiorgio Giacchè, per i materiali di studio e i ricordi personali con cui hanno supportato la presente indagine. Il titolo ricalca quello di un precedente contributo analitico di Marco Angius dedicato a Salvatore Sciarrino: Sui poemi concentrici. Da Sciarrino a Dante (con un'apparizione di Carmelo Bene) (Angius 2002: 50-56).

I. Lectura Dantis, voce recitante Carmelo Bene, musiche di Salvatore Sciarrino, musicista solista David Bellugi; fonici: G. Burroni, M. Contini, B. Bucciarelli; regia mixer: Carmelo Bene; produzione: Rino Maenza. Bologna, Torre degli Asinelli, 3r luglio I98I.

2. Alla figura di Carmelo Bene (1937-2002), attore, regista, drammaturgo, filosofo, scrittore e poeta italiano, è dedicata una vasta bibliografia, tra i cui titoli si segnalano: Grande 1982; Fofi, Giacchè 1995; Giacchè 2007. Sul percorso artistico e di vita di Carmelo Bene cfr. Bene, Dotto 1998.

3. L'idea di affrontare Lectura Dantis dal versante della musica e non da quello del teatro si deve in particolar modo alla lettura di due scritti dedicati a Carmelo Bene: la già citata monografia Antropologia di una macchina attoriale di Piergiorgio Giacchè e l'articolo Un muezzin per Dante di Andrea Cortellessa che, pur se in modo diverso - il primo ripercorrendo l'intera produzionedi Bene, il secondo concentrandosi sulla registrazione sonora di Lectura Dantis -, ravvisano nelle qualità soniche delle perfomances di Bene alcuni dei cardini del suo essere "dentro e contro" l'arte della scena. Per questa ragione gli scritti di Giacchè e Cortellessa ritorneranno più volte nel corso della presente indagine, citati in qualità di supporti fondanti per un avvicinamento ad un esempio significativo di teatro "sonoro" beniano.

4. Sul Principio audiotattile cfr. anche Caporaletti 2000.

5. Cfr. McLuhan I962 e 1964; McLuhan, McLuhan 1988; De Kerckhove I99I. 
della ricezione dei messaggi; ad essere posta in rilievo è "la funzione dinamica che i principi epistemici inglobati nell'assetto mediale, attraverso cui i costrutti culturali sono prodotti e comunicati, esplicano sulle valenze simboliche dei costrutti stessi, oltre che sulla riconfigurazione dell'assetto percettivo e sugli schemi concettuali di chi li produce e recepisce" (Caporaletti 20IO: 5I-52). Bisogna infine sottolineare che la specificità del principio audiotattile si manifesta compiutamente nella fattività della produzione del testo in presenza, anziché della riproduzione musicale esecutiva di un testo creato in assenza: il principio audiotattile "ha la sua magnificazione nel momento in cui per la comunicazione musicale si passa da un regime allografico, quello della notazione, che presuppone una diversificazione di ruoli tra compositore ed esecutore, a quello autografico, in cui sono poi embricati, attraverso la medialità tecnologica, i processi che definisco di codifica neoauratica" (Caporaletti 20I0: 54). Con codificazione neoauratica Caporaletti intende una concezione antinomica a quella della perdita dell'aura per l'opera d'arte nell'era della riproducibilità tecnica, così come teorizzata da Walter Benjamin nel 1936 (Benjamin 20I4: 19-56). Se è infatti indubitabile che con la replicabilità tecnologica si debba rinunciare all'unicità dell'opera dal vivo, è altrettanto vero che "gli aspetti riconducibili al principio audiotattile trovano nella registrazione sonora il mezzo per una fissazione di alcuni indici significativi delle qualità processuali/evenemenziali, che ricostituiscono per queste formazioni musicali un nuovo modello di auraticità attraverso il supporto tecnologico" (Caporaletti 20I0: 54).

Alla luce di questa premessa, nel corso della disamina che segue si osserverà come il principio audiotattile (che si propone come modello cognitivo), attraverso la codifica neoauratica (la registrazione sonora), inviti a una scoperta dei valori che attraversano un processo creativo come quello rappresentato da Lectura Dantis di Carmelo Bene, restituendo una lettura profonda di un fenomeno complesso che nasce dall'intersezione di commemorazione pubblica, perpetuarsi di un canone della letteratura italiana, performance scenica, phoné, musica (composta, montata, amplificata), e per questo difficilmente categorizzabile.

\section{Bologna, Torre degli Asinelli, 3I luglio i98I}

Prese a soffiare un vento di scirocco che fastidiava non poco li megafoni issati in su le lance. Io mi forzavo trattener gli spirti e darmi pace, come usa pugilatore avanti la sua tenzone.

Era l'ora. M'inerpicai sui pioli d'una impervia scaletta e finalmente mi mostrai alla folla che, meravigliata forse più di un suo numero che del miraggio mio, salutò in me l'attesa. Fu di plauso un boato indescrivibile che si ripercoteva dalle piazze lontane e ne le strade adiacenti tutte. 
Apparvi. Li occhi mia chiusi al leggio luminescente, presi a cantar li versi d'Allegheri. Ma d'altrove, nel tempo delle nevi e del vin cotto di mia parvola vita. E venni meno, e il canto seguitò come profferto da ser Boccaccio in quella villa istessa settecent'anni prima. Li suoni rincorreansi sovra i tetti, e il silenzio divoto de le genti omai fatte incantamento mi suase al dolce vanire (Bene 2005: 76-77).

Così Carmelo Bene ricordava la sua Lectura Dantis dalla Torre degli Asinelli, avvenuta il 3I luglio 198I, nell'anniversario della Strage di Bologna. ${ }^{6}$ L'occasione commemorativa - non trascurabile nell'analisi della performance - fu accompagnata da numerose polemiche: come testimoniano i quotidiani dell'epoca, ${ }^{7}$ la scelta del sindaco Renato Zangheri $^{8}$ di affidare a Bene l'orazione in ricordo delle vittime dell'attentato suscitò diversi malumori, al punto che alcuni esponenti del consiglio comunale non esitarono a definirla "una pagliacciata", mentre il socialista Massimo Pini, membro del consiglio d'amministrazione Rai, bocciò il progetto delle riprese televisive dell'evento. Al contrario si trattò, come ricordano numerosi testimoni che vi presero parte, di una serata auratica (Cortellessa 2004): ${ }^{9}$ lo stesso Bene individua nel suo

6. La strage colpì la stazione ferroviaria di Bologna la mattina di sabato 2 agosto 1980; fu uno degli atti terroristici più gravi avvenuti in Italia nel secondo dopoguerra e al contempo uno degli ultimi atti della strategia della tensione che sconvolse la nazione negli anni Settanta. Nell'attentato rimasero uccise 85 persone ed oltre 200 furono ferite. Come esecutori materiali la magistratura ha condannato alcuni militanti di estrema destra, appartenenti ai Nuclei Armati Rivoluzionari (NAR), Giuseppe Valerio Fioravanti e Francesca Mambro. I mandanti sono tuttora sconosciuti.

7. Si propone di seguito una selezione dei numerosi articoli apparsi sui quotidiani nei giorni precedenti e seguenti alla Lectura Dantis: S. Colomba, "Carmelo Bene: 'Io verrò in ogni caso, chi non mi capisce è un provinciale”, Il Resto del Carlino, 5 luglio I98I; "Il programma uscirà indenne dalla bufera delle polemiche?", Il Resto del Carlino, 8 luglio I98I; R. Palazzi, "Ma questi milioni sono per Bene?", Il Corriere della Sera, 22 luglio I98I; V. Monti, "Alla vigilia della recita Bene polemizza su giovani e terrorismo", Corriere della sera, 3I luglio I98I; F. Pezzato, S. Colomba, "Un Carmelo Bene 'teologico' al di sopra delle polemiche", Il Resto del Carlino, 3r luglio 1981; Goldoni L., "Dante Best-seller con il prof. Bene", Corriere della Sera, 2 agosto I98I; C. Marabini, "Dante sull'Asinelli: ha vinto la poesia", Il Resto del Carlino, 2 agosto 1981; M. Scialoja, "Celebrazioni Bolognesi - 'La Merda', poema di Carmelo Alighieri”, L'Espresso, 2 agosto $198 \mathrm{r}$.

8. Così Rino Maenza ricorda la nascita della commissione: "La Lectura Dantis a Bologna nacque da una passeggiata notturna di un dopo teatro, quando il sindaco Zangheri chiese a Carmelo: 'Perché non fai qualcosa che ricordi laicamente la strage della stazione?' E discutendo, ragionando così, venne fuori: 'Ecco, bisognerebbe fare una riflessione laica, recuperare la Bologna di Dante” (cfr. Maenza 20I2: 223). La storia del forte legame di Carmelo Bene con Bologna risale a molto tempo prima del caso Lectura Dantis: nei primi anni Sessanta, infatti, Bene era ancora un giovane sperimentatore del teatro d'avanguardia e l'allora assessore alla cultura provinciale Carlo Maria Badini lo ospitò nel piccolo teatro della Ribalta. Il suo primo spettacolo da autore fu un evento unico: Majakovskij. Quattro diversi modi di morire in versi, un'antologia di alcune pagine poetiche di Majakovskij, Esenin, Pasternak e Blok. Una sola recita, che andò in scena il 26 gennaio 1960, lo vide sul palco accompagnato al pianoforte da Sylvano Bussotti, il quale aveva composto musiche originali per lo spettacolo. Fu questa l'occasione per l'inizio di un fortunato e duraturo rapporto artistico tra Carmelo Bene e le istituzioni bolognesi.

9. L'articolo è stato scritto da Cortellessa - presente la sera del 2 agosto I98I a Bologna - in 
Dante "uno dei più infernali casini del dopoguerra ma anche il più grande, irripetibile evento della mia vita" (Bene e Dotto 1998: 242). Le pagine relative, in Sono apparso alla Madonna, fanno da contraltare mistico, quasi sciamanico, scritto in dannunziano pastiche quattrocentesco, a una vicenda quanto mai terrena. La sera del 3r luglio, Carmelo Bene, salutato da una folla immensa, arrampicatosi su una scaletta, si installò come un vate in cima alla Torre degli Asinelli, ${ }^{\text {Io }}$ nel cuore di Bologna; si dispose, dietro al leggio illuminato, a strapiombo sulla gente ai suoi piedi (centocinquantamila circa, gli spettatori), e cominciò la Lectura Dantis.

Anche chi non aveva mai letto Dante quella sera si emozionò. Il dolore e la speranza della Commedia si mescolarono al dolore per i morti e alla speranza per i feriti, vissuti dalla città dal giorno dell'orrenda strage. Il silenzio di quella sera permise di rivivere il dramma del 2 agosto. [...]

Non era facile il silenzio, quella sera del $3 \mathrm{I}$ luglio. La rabbia era ancora una brace accesa. Le Brigate rosse cercarono di trasformarla in rivolta armata. Poco prima della Lectura Dantis furono trovati volantini che incitavano a costruire una "colonna armata bolognese" e un appello registrato fu diffuso da un'auto con altoparlante. Il silenzio delle migliaia di via Rizzoli fu una risposta. Bologna voleva giustizia, non vendette (Meletti 20IO).

Bene scelse di proporre alla città un'orazione civile che si configurasse al contempo come "apparizione": il luogo prescelto non fu un teatro, una piazza, un grande spazio all'aperto; posizionandosi sulla cima di una torre, Bene invitò i cittadini ad alzare gli occhi al cielo, ad assistere ad un'epifania e a perdersi nel suono dei versi di Dante, propagati sopra i tetti e lungo le strade della città da un gigantesco impianto di amplificazione. L'effetto generato fu simile a quello che si può udire quando il muezzin richiama i fedeli alla preghiera (Cortellessa 2004), con la differenza che mentre il muezzin, dal minareto, modula secondo una cantilena la formula stabilita, Bene, dalla Torre, intonò un canto alla ricerca di continue variazioni e differenti soluzioni.

I pochi elementi sin qui richiamati all'attenzione consentono di affermare che non si può ridurre la performance del 3I luglio a mero spettacolo di recitazione: Lectura Dantis va analizzata in quanto non solo teatro, ma soprattutto "esibizione canora", pur nella coscienza che l'esplicitazione del cantare resta in Bene quasi sempre "sottocutanea" ma non "implicita".

occasione della ristampa della registrazione audio di Lectura Dantis, pubblicata da Luca Sossella Editore.

Io. Le due torri, Asinelli (la maggiore) e Garisenda (la minore e più pendente), sono il simbolo della città di Bologna; entrambe pendenti, sono situate al centro di una raggiera, non particolarmente ampia, da cui si dipartono le principali strade cittadine. La Torre degli Asinelli è alta 97,2 metri; la Garisenda 48 (citata più volte da Dante, nella Divina Commedia e nelle Rime, a riprova del suo soggiorno a Bologna). 


\section{RECitAR CANTANDO, O VICEVERSA}

Nel corso degli anni Settanta, Bene porta avanti un'esasperata linea di ricerca che lo conduce alla costruzione scenica dell'assenza; il suo teatro "oppone alla festa dell'energia - ancora attraente e plausibile per i consumatori onnivori dello spettacolo-merce - la sua stessa decadenza" (Giacchè 2007: I23): lo spettacolo dell'assenza si fa assenza dello spettacolo, esempio magico e arcaico di meraviglia. Facendo riferimento ai lavori di quegli anni si potrebbero ricordare le minuzie di centinaia di micro-movimenti, semitoni smorzati, espressioni quasi cancellate, abiti cambiati, chiusure improvvise, cesure continue, suoni spinti a morire all'acuto o al grave, gesti ripiegati sul nascere, parole liquefatte mentre sono ancora in gola: la costante alla quale si assiste e che si ode è la sparizione.

Momento di transizione fondamentale è S.A.D.E., spettacolo del 1975 , ancora teatrale, in cui le macchine del suono e il suono delle macchine hanno un peso non più periferico e un ruolo non soltanto accessorio (Giacchè 2007: I24-I26): quella di S.A.D.E. è una musica del caos, che esce dai ranghi per non farvi più ritorno. Il primato del suono, spirito guida della successiva ricerca oltre-teatrale di Bene, diventa esplicito e conduce in un luogo inesplorato, che è al contempo molto lontano tanto dal canzoniere brechtiano quanto dal varietà. Il teatro di Bene della fine degli anni Settanta, scrive Piergiorgio Giacchè, "funziona come un'opera invertita, con stacchi musicali che non fanno da sottofondo ma che invece sfondano le parole e le trasformano in note e, dall'altra, con battute recitate che - da qualunque testo provengano si distendono fin dal principio almeno in versi e diventano alla fin fine per lo meno suoni" (Giacchè 2007: I28). Per Bene la musica è come un continente vasto che è insensato ridurre ad aggettivo di un genere teatrale; al contrario il teatro può spiegarsi soltanto all'interno della musica, e anche se non ne diventerà mai del tutto parte, può sopravvivere solo all'interno dei suoi confini.

Il teatro dell'assenza, a cui Bene mira sempre più a partire dagli anni Settanta, non può che essere abitato da un unico invisibile protagonista: la voce (Giacchè 2007: 130). Essa è destinata a impersonare il vuoto e ad assorbire in sé il corpo dell'attore, la materialità della scena, i frammenti del testo. Nella voce l'arte scenica può regredire fino alla sua natura primigenia, liberandosi delle convenzioni e delle costrizioni dei significati e delle forme. La centralità restituita alla voce trasforma gli spettacoli di Bene in "concerti": dimenticare che quella voce nasce e si afferma come protagonista dello "spettacolo della dissoluzione" del teatro, significherebbe però tradire Bene. L'utilizzo della voce che Bene fa, non è il risultato di una studiata riforma della recitazione, ma, al contrario, un cantare che si soffoca nel dire, perché la musica è la matrice e non solo l'obiettivo della sua recitazione. 
Gli spettacoli-concerto Manfred e Lectura Dantis si configurano come il punto d'arrivo di un percorso di dissoluzione teatrale cominciato alcuni anni addietro, e si caratterizzano per il modo unico di "cantar recitando" (Giacchè 2007) - fatto di invenzioni, variazioni e continui cambi di intonazione - che Bene mette in atto. Egli inaugura una via originale che trasforma la questione del rapporto tra cantare e recitare; si ha l'impressione, ascoltando la registrazione di Lectura Dantis, che dopo Bene la relazione tra teatro e musica sia così profondamente trasformata che addirittura manchino gli strumenti terminologici per ridefinirla. Lo stesso Deleuze, ad esempio, che si era limitato a paragonare il "recitar cantando" del Bene degli anni Settanta allo Sprechgesang, dopo il Manfred si avvide che il tentativo operato non era quello di sovrapporre la recitazione al canto, bensì di ricavare dalla voce forze sonore che tuttavia non si confondessero col canto: "queste nuove potenze potranno accompagnare il canto, cospirare con esso, ma non formeranno né un canto e neppure uno Sprechgesang, è l'invenzione di una voce modalizzata o piuttosto filtrata. [...] Si tratta al contempo di fissare, creare o modificare il colore di base di un suono (o di un insieme di suoni), e di farlo variare o evolvere nel tempo, di cambiarne la curva fisiologica" (Deleuze in Bene 198r: 8).

In Lectura Dantis, ancor più che in Manfred, grazie all'imponente sistema di amplificazione utilizzato per diffondere la performance nelle strade circostanti alla Torre degli Asinelli, ${ }^{\text {II }}$ ogni verso o singola parola "recitata" sembra allungarsi e riverberare, per dar luogo a virtuosi effetti di suono che sono al contempo ricercati difetti di senso che "manomettono" il significato del testo dantesco. Attraverso la mediazione degli amplificatori e del microfono, la voce di Bene si fa "totale", attraversando anche chi non è direttamente sotto la Torre: la voce, allo stesso tempo corpo e strumento, affranca l'attore dal corpo stesso. ${ }^{12}$ Il corpo-attore di Carmelo Bene che legge dall'alto, appena visibile, la Divina Commedia, inventa e restituisce alla moltitudine degli

II. Così Carmelo Bene descrive in Sono apparso alla Madonna gli stregati marchingegni, affidati alle cure di Rino Maenza, utilizzati per amplificare la Lectura Dantis: "In verità, trattavasi d'assai complessi ponte vociatore che permetteva ascoltare e interloquire con la bottega ubicata in quel di via delle calzolerie e di là con gli spalti della Torre Asinelli fermentata dai genieri del suono in bel travaglio insolato che [...] esperimentavano i tuoni e lor colori e percezione e potenza d'uopo, ché la sera scendeva e n'era forza sbaragliar le nemiche fazioni, e, d'altro canto, produrre grande strazio di core ne le genti in ascolto. Questi genieri scelti in su li spalti roventi del meriggio, intimoriti punto del gran tumulto de le folle in basso, con ordigno predisposto intendevano li consigli del mastro in succitata bottega Maenza Salvator nomato, non vedente d'occhi due e perciò abilissimo conoscitor de la musica e inventor di congegni altoparlanti di provata efficacia atti a disperdere qual si sia resistenza dialettica inimica con l'assordar de li timpani se in caso necessitade" (Bene 2005: 76-77).

I2. Robert Murray Schafer ha coniato il termine "schizofonia" riferendosi alla dicotomia tra il suono originale e la sua riproduzione elettroacustica. I suoni originali sono sempre connessi con il meccanismo che li produce. I suoni elettroacustici invece sono copie che possono essere riprodotte in tempi e luoghi diversi. 
ascoltatori, con un significato nuovo, una possibilità di ascolto mai sperimentata prima. La voce, che raggiunge anche chi in lontananza non sa cosa stia accadendo, suscita stupore e fascinazione quasi mistiche, infernali e paradisiache al contempo, proprio grazie all'invisibilità della sorgente. La voce filtrata dall'impianto d'amplificazione, strumento musicale assoluto, realizza la comunicazione "da un di dentro" - quello del performer Carmelo Bene ad un altro "di dentro" quello del pubblico: "ecco il di dentro che trapassa a un altro di dentro. Voce-ascolto e voce-udita, intime proprio perché dislocate, sottratte l'una all'altra. [...] Chi parla e chi ascolta sono dissociati in natura e riassociati in tecnologia" (Bene e Dotto 1998: 337-338). E ancora, come precisato da Deleuze, "non è questo o quel personaggio che parla, ma il suono stesso diventa personaggio, quel preciso essere sonoro diventa personaggio" (Deleuze in Costa 2003). L'impressione generata è quella di trovarsi di fronte a una sovrapposizione di generi; prendendo in prestito le parole utilizzate da Gerardo Guarnieri nel recensire Faust-Marlowe-Burlesque, Bene è superbo "nel didattico come nell'intimo, nel pop infernale come nel sardonico affettuoso; è satrapico e orientale; la sua recitazione speziata nasillarde, eccelle nel tono pseudo confidenziale, nel condiscendente dileggiante, nello pseudoieratico, nell'erotico-onanistico, nel blasfemo sarcastico: che antologia, che ventaglio" (Guarnieri 1993: 295).

Come nel caso di molte musiche che si basano sul principio dell'improvvisazione, così per Lectura Dantis e altri spettacoli-concerto di Carmelo Bene, si potrebbe affermare che ci sono opere che appartengono solo a chi le ha "performate", data la non riconducibilità di queste opere d'arte al solo testo che vi sta alla base. In una ipotetica successiva riedizione di uno spettacolo come Lectura Dantis, un attore-cantante si troverebbe a fare i conti con un solo possibile modello di cantar recitando - quello di Bene - fuori dal quale non si darebbe più lo stesso dissonare del senso, non si perverrebbe più allo stesso grado di ambiguità, non si cadrebbe nello stesso equivoco, non si suggerirebbe più la stessa sensazione. Bene, eccedendo le forme e tradendo i significati in ogni pur minimo segmento o momento della sua recitazione, impedisce ogni possibilità di riproduzione della sua performance, nonostante l'apparente facilità imitativa che i suoi toni e gesti vocali lascerebbero presupporre (Giacchè 2007: I3I-I32). La registrazione sonora dell'evento è una chiara testimonianza di quanto affermato. ${ }^{13}$

I canti danteschi, terzine incatenate d'endecasillabi in volgare fiorentino, potrebbero considerarsi al pari di una rigida partitura musicale che va eseguita,

13. La registrazione live di Lectura Dantis è stata edita nel I98I da CGD, LP 20283, poi ristampata in Cd audio a cura di Luca Sossella Editore (Roma) nel 2004. Esiste anche una registrazione video della Lectura Dantis dal titolo Carmelo Bene legge Dante, a cura di Rino Maenza, Dvd, Marsilio, Venezia 2007. 
ma per Bene leggere, riferire e dire un testo significa "dimenticarlo". L'attore, se si preoccupa della memoria, non può lasciare spazio e densità al delirio della voce. E dimenticando, Bene porta avanti un processo che non è lontano dall'idea di estemporizzazione, cardine delle musiche audiotattili (Caporaletti 2005: 98-II5). Tuttavia la precisa selezione di alcuni testi della Divina Commedia e la consapevolezza di una registrazione live comportano che, nel momento in cui la registrazione prende vita, si realizza un testo, fissato e delineato dalle sue intrinseche strutture formali, punto di riferimento per quanti successivamente si avvicineranno all'opera dantesca.

Cancellando il dogma della dimenticanza, che Bene impone alla lettura, le interiezioni e le interruzioni, le accelerazioni e le sospensioni, le altisonanze e gli sprofondamenti che animano il testo finirebbero col ruotare intorno al solo concetto d'interpretazione occidentale. La rielaborazione personale di Bene della Divina Commedia si compone invece dell'incessante ed esigente inventio e selezione dell'auctor e della precisione e della capacità di estemporizzare dell'actor (Giacchè 2007: 132), cristallizzandosi attraverso la registrazione sonora in opera d'arte. Proprio come avviene nel caso delle musiche di tradizione orale, Bene non elude del tutto le prescrizioni che gli sono poste dal testo, ma lo flette fino all'estremo, scalfendone quei valori che gli erano stati irrevocabilmente attribuiti da una tradizione secolare (Caporaletti 2005: I4-I6).

Il nuovo testo, inscritto sul supporto tecnologico di riproduzione fonografica, viene così sottratto all'evanescenza della celebrazione del luglio del I98I e proiettato in un'oggettiva sfera di referenzialità come oggetto estetico.

In Lectura Dantis la voce di Bene è infine medium tra il corpo dell'attore e lo sguardo dello spettatore, assumendo in sé, oltre ai significati e ai significanti del testo, il repertorio della gestualità. La voce annulla in parte i valori tradizionali affidati al testo di Dante e restituisce un corpo fisico alle immagini mentali, obbligandole a un percorso uditivo sempre nuovo: un baccanale sensitivo che lo spettatore riceve come volume sonoro in continua mutazione e che il suo occhio è chiamato a riprodurre. La voce sola trattiene in sé e rimanda l'intreccio, le situazioni, i conflitti di uno o più personaggi; si traveste nella varietà dei sensi che le sono offerti: la sua capacità di estemporizzare, dando corpo e anima allo scritto dei versi danteschi, rende il suo agire attraverso l'amplificazione "pre-vedibile" agli occhi dello spettatore in ascolto.

\section{3. “...Il suono stesso diventa personaggio"}

Significativa è la selezione dei canti della Divina Commedia compiuta da Bene: egli sceglie frammenti dal V, XXVI e XXXIII dell'Inferno, dal VI e dall'VIII del Purgatorio e dal XXIII, XXVII e VII del Paradiso, ai quale aggiunge due sonetti dalle Rime e dalla Vita Nova, "Guido, i' vorrei che te e Lapo ed io" $e$ 
"Tanto gentile e onesta pare". Paolo e Francesca (If. V), Ulisse (If. XXVI), Ugolino (If. XXXIII), Sordello (Pg. VI), "l'ora che volge il disio" (Pg. VIII), “i pleniluni sereni” ( $P d$. XXIII), i due sonetti: si tratta di alcuni dei passi più noti della produzione dantesca - con l'eccezione delle due meno conosciute visioni paradisiache (l'invettiva di San Pietro contro i suoi successori del canto XXVII, e l'intonazione di lode a Dio di Giustiniano nel VII canto) - anche perché cardini della formazione scolastica italiana di base. Tale scelta amplifica la volontà di Bene di produrre una lettura come "non ricordo": filtrata dalla voce, una storia - anche la più conosciuta - con il suo dramma, riappare in forma di eco al quadrato (perché il filtro è doppio, dal momento che la voce passa attraverso il microfono).

Alla fluidità del narrare Bene sostituisce gli sprazzi e i singulti di un cantar recitando che a tratti sembra ricordare quello delle prefiche, preferendo al racconto delle gesta l'evocazione delle sembianze, e alla restituzione della vicenda lo smembramento della stessa. Quello compiuto da Bene non va però confuso con un semplice esercizio di stile: trapassati nella voce, i versi e i loro significati riemergono mutati e, disimpegnati dall'obbligo della comunicazione, si trasfigurano oltre la comune rappresentazione attribuita loro nei secoli. L'intento di Bene - contrariamente a quello che potrebbe cogliere un ascoltatore disattento - è quello di restituire al testo quella densità e credibilità smarrite a causa delle troppe meccaniche riproposizioni dello stesso. La voce che avvolge o che mangia le parole non va verso una forma di canto evanescente, futile e compiaciuta; si spinge piuttosto verso la riconquista del mistero stesso della parola.

Decisiva è la scansione delle letture, che mostra quanto Bene riesca a incidere sul tempo della recitazione e del canto, deframmentando e alterando il ritmo di una lettura che, data la struttura incatenata delle terzine, tenderebbe a conservare una forte coesione ritmica.

Bene dà voce alla topica e veemente tensione invettivale che permea lunghi tratti della Commedia (Cortellessa 2004): formidabili i suoni aspri e chiocci della parlata di San Pietro (la triplice esclamazione "il luogo mio") e il sibilare sferzante delle rime in -aca, -esse e -aschi ( $P d$. XXVII),

La provedenza, che quivi comparte vice e officio, nel beato coro silenzioso posto avea da ogne parte, quand'io udi': "Se io mi trascoloro, non ti maravigliar, ché, dicen'io, vedrai trascolorar tutti costoro.

Quelli c'usurpa in terra il luogo mio, il luogo mio, il luogo mio che vaca nella presenza del Figliuol di Dio, 
fatt' ha del cimitero mia cloaca del sangue e della puzza; onde 'l perverso che cadde di qua sù, la giù si placa".

"Non fu la sposa di Cristo allevata del sangue mio, di Lin, di quel di Cleto, per essere ad acuisto d'oro usata;

ma per acquisto d'esto viver lieto e Sisto e Pio e Calisto e Urbano sparser lo sangue dopo molto fleto.

Non fu nostra intenzion ch'a destra mano d'i nostri successor parte sedesse, parte da l'altra del popol cristiano;

né che le chiavi che mi fuor concesse, divenisser signaculo in vessillo che contra battezzati combattesse; né ch'io fossi figura di sigillo a privilegi venduti e mendaci, ond'io sovente arrosso e disfavillo.

In vesta di pastor lupi rapaci si veggion di qua sù per tutti i paschi: o difesa di Dio, perché pur giaci?

Del sangue nostro Caorsini e Guaschi S'apprecchian di bere: o buon principio, a che vil fine convien che tu caschi! ${ }^{I 4}$ (Pd. XXVII i6-27, 40-60)

Pietro, dopo aver anticipato l'aspetto che presto invaderà tutto il cielo, aumentando l'attesa di Dante e dando solennità universale alle sue parole, rivolge una sferzante invettiva contro Bonifacio VIII indegno usurpatore, in quanto Cristo non guida misticamente la sua Chiesa attraverso di lui e non lo riconosce come suo vicario. Il canto esibisce un allestimento di motivi tematici che preannunciano l'immediato futuro narrativo dell'opera ed allo stesso tempo costituiscono una chiusura rivolta al già trascorso; con le sue ritualità interne il canto descrive un passaggio definitivo del poema, quello tra terra e cielo, tra penitenza e beatitudine, tra sofferenza e felicità, che Bene scandisce attraverso coordinate musicali gravi e sferzanti modalità intonative, in una perfetta soluzione di continuità tra rappresentazione musicale e poetica che precede qualsiasi considerazione sulla terribilità di quanto viene descritto.

I4. Il secondo discorso di Pietro dichiara i vizi per cui il posto del vicario di Cristo è ora "usurpato", e lo fa attraverso la forma retorica delle negazioni. Pietro confronta la Chiesa di un tempo con quella presente: ai martiri di un tempo si sono sostituiti papi che dividono il popolo cristiano. Quelle chiavi che un tempo furono date a Pietro per salvare, servono ora per portare guerra e vendere privilegi immotivati, al punto che guardando dal cielo la terra, si scorgono in tutte le regioni del mondo abitate dal gregge di Cristo lupi famelici travestiti da pastori. 
Quindi Bene riproduce gli eccezionali e improvvisi vortici sonori di Ulisse nel XXVI canto dell'Inferno. Dopo la nota esortazione "fatti non foste a viver come bruti, / ma per seguir virtute e canoscenza", con cui Ulisse ha penetrato i cuori dei suoi compagni, convincendoli a solcare nuovamente le onde del mare alla scoperta del mondo sconosciuto, prosegue la descrizione del rischioso e arduo viaggio, sino alla tragica conclusione del canto: il gorgo finale che inabissa la nave oramai giunta di fronte all'alta cima del Purgatorio.

Li miei compagni fec'io sì aguti, con questa orazion picciola, al cammino, che a pena poscia li avrei ritenuti; e volta nostra poppa nel mattino, de' remi facemmo ali al folle volo, sempre acquistando dal lato mancino.

Tutte le stesse già de l'altro polo Vedea la notte e 'l nostro tanto basso, che non surgea fuor del marin suolo.

Cinque volte racceso e tante casso lo lume era di sotto da la luna, poi che 'ntrati eravam ne l'alto passo, quando n'apparve una montagna, bruna per la distanza, e parvemi alta tanto quanto veduta non avea alcuna.

Noi ci allegrammo, e tosto tornò in pianto, ché de la nuova terra un turbo nacque, e percosse del legno il primo canto.

Tre volte il fé girar con tutte l'acque; a la quarta levar la poppa in suso e la prora ire in giù, com'altrui piacque, infin che 'l mar fu sovra noi richiuso". (If. XXVI I2I-I42)

E ancora si sofferma su momenti di poetica meraviglia, quale, ad esempio, il dileguarsi fino a svanire delle parole in legato nell'Osanna estatico del VII canto del Paradiso. Il canto si apre con un inno di lode a Dio, cantato da Giustiniano al termine del suo discorso (riportato nel V canto del Paradiso); la solennità dell'apertura in lingua latina liturgica prelude al grande tema teologico che il canto tratterà, ma che Bene decide di elidere, chiudendo con le terzine di rara bellezza 7-9, IO-I2 e I3-I5: esse esprimono un profondo incanto, quasi un rapimento dell'animo, ripetuto con versi di insistente dolcezza. $\grave{E}$ questo uno dei momenti in cui, nell'ascesa al Paradiso, tra le visioni e le parole si pone una sosta di segno mistico, quasi a punteggiare il percorso con un richiamo costante alla misteriosa ultima realtà a cui esso è diretto. E così come le anime svaniscono agli occhi di Dante, per l'improvvisa distanza posta tra loro e il poeta dal rapido allontanarsi delle anime stesse, così Bene lascia 
andare il ricordo dei defunti della Strage di Bologna, prendendo in prestito l'immagine dantesca per concludere l'orazione funebre.

"Osanna, sanctus Deus sabaòth, superillustrans claritate tua felices ignes horum malacòth!".

Così, volgendosi a la nota sua, fu viso a me cantare essa sustanza, sopra la qual doppio lume s'addua; ed essa e l'altre mossero a sua danza, e quasi velocissime faville mi si velar di sùbita distanza.

Io dubitaba e dicea 'Dille, dille'! fra me, 'dille' dicea, 'a la mia donna che mi diseta con le dolci stille'. Ma quella reverenza che s'indonna Di tutto me, pur per Be e per ice, mi richinava come l'uom ch'assonna. (Pd. VII I-I5)

Infine Bene, nel finale del $\mathrm{V}$ canto dell' Inferno, indaga il tempo della commozione, scandito non dalla voce, bensì dalle pause e dai silenzi:

Mentre che l'uno spirto questo disse, l'altro piangea; sì che di pietade io venni men così com'io morisse.

E caddi come corpo morto cade. (If. V I39-I42)

Il significato dello svenimento di Dante va raccolto da tutto l'andamento della storia e dell'incontro con Paolo e Francesca narrato nel V canto dell' Inferno, e non può essere ridotto a semplice commozione per una singola tragica vicenda; è questo uno dei punti in cui la pietà dantesca giunge ad un grado di intensità eccezionale, elevandosi a comprensione di tutta la condizione umana, attraverso versi che esprimono tutta la fatalità di quella caduta. Ed è in virtù di questa intensità eccezionale - che va oltre la semplice vicenda di Paolo e Francesca - che Bene sceglie il V canto dell'Inferno per aprire la Lectura Dantis del 2 agosto del I98I.

Attraverso l'utilizzo di spettrogrammi è possibile esemplificare alcune delle variabili del percorso sonoro proposto da Bene, riportando graficamente gli esempi citati: i picchi similari raggiunti e mantenuti nella triplice rima in -aca del XXVII canto del Paradiso (Es. I); il gorgo che la voce genera nel finale del XXVI canto dell' Inferno prima di cadere ripetutamente (Es. 2); il legato tenuto sulla stessa altezza dell' incipit dell' Osanna del VII canto del Paradiso (Es. 3); le ripetute e asimmetriche pause che scandiscono gli ultimi versi del V canto dell'Inferno (Es. 4). 


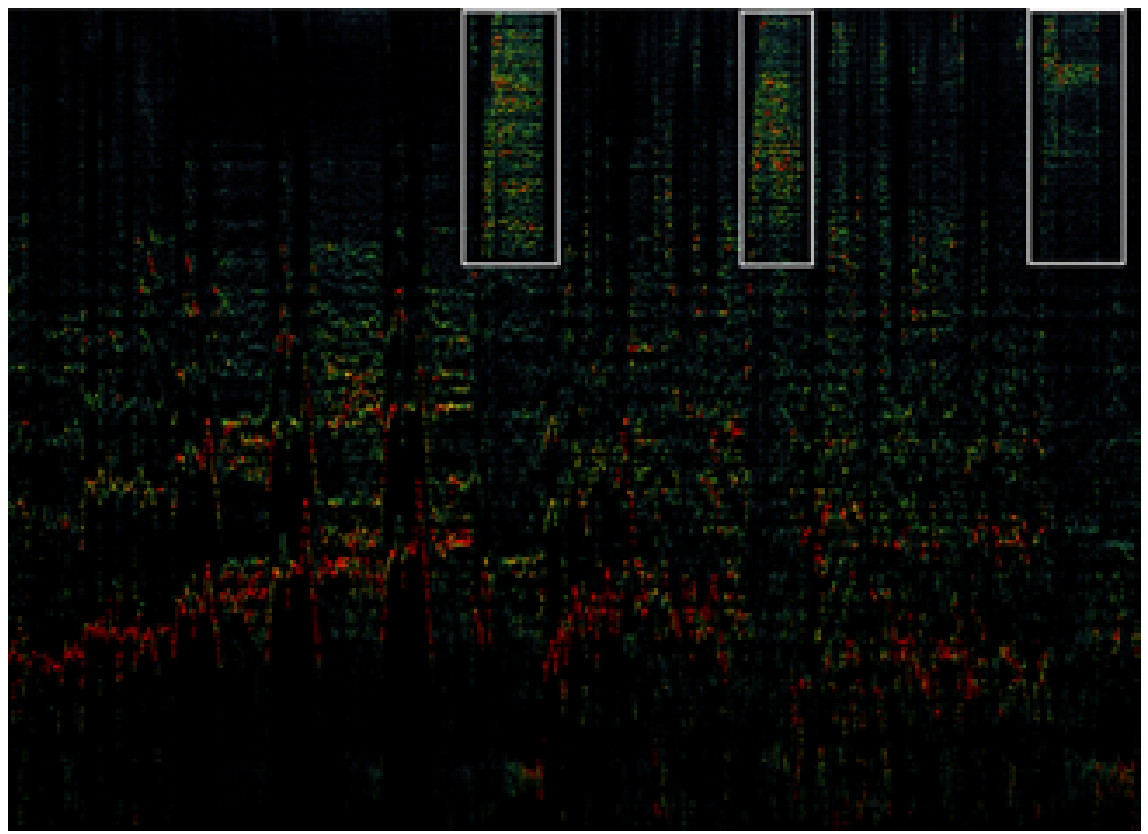

Es. I. Pd. XXVII I6-27. Spettogramma, peak frequency (riquadro: rime in -aca, vv. 23, 25 e 27).

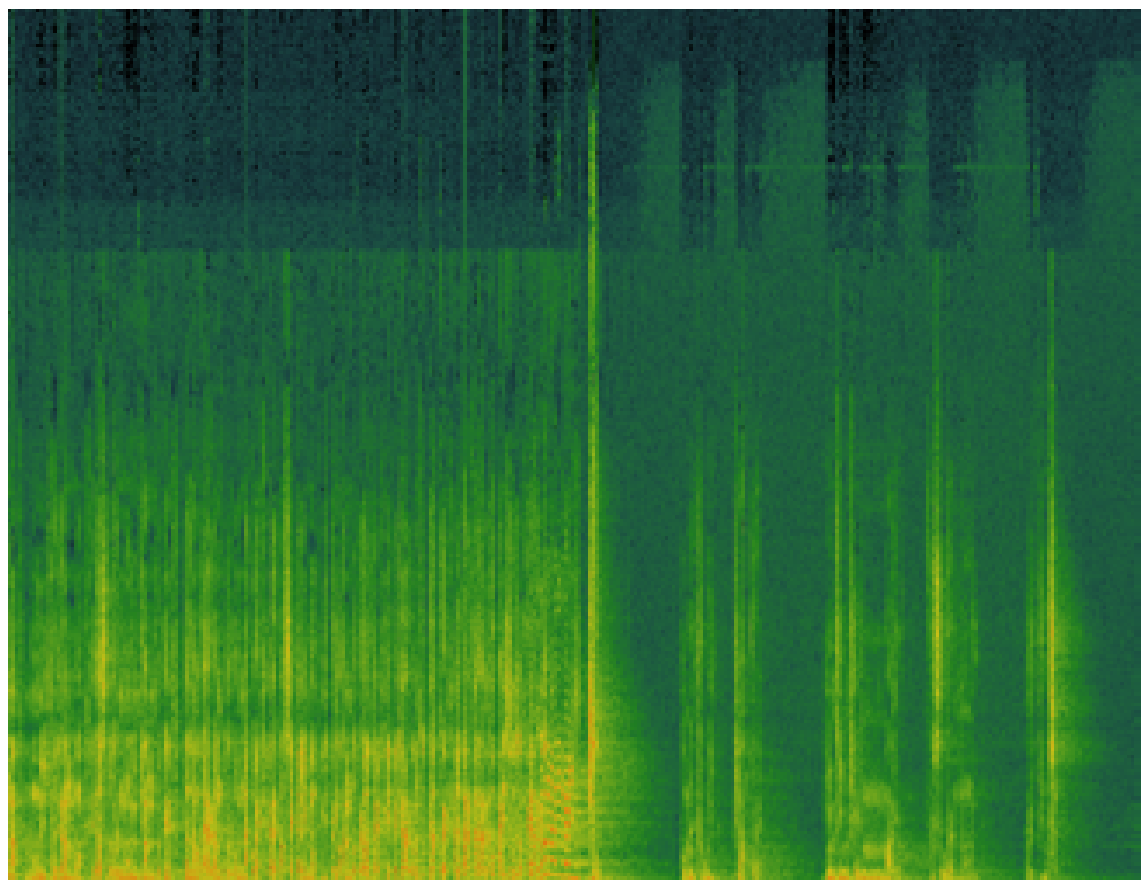

Es. 2. If. XXVI 136-I42. Spettogramma. 


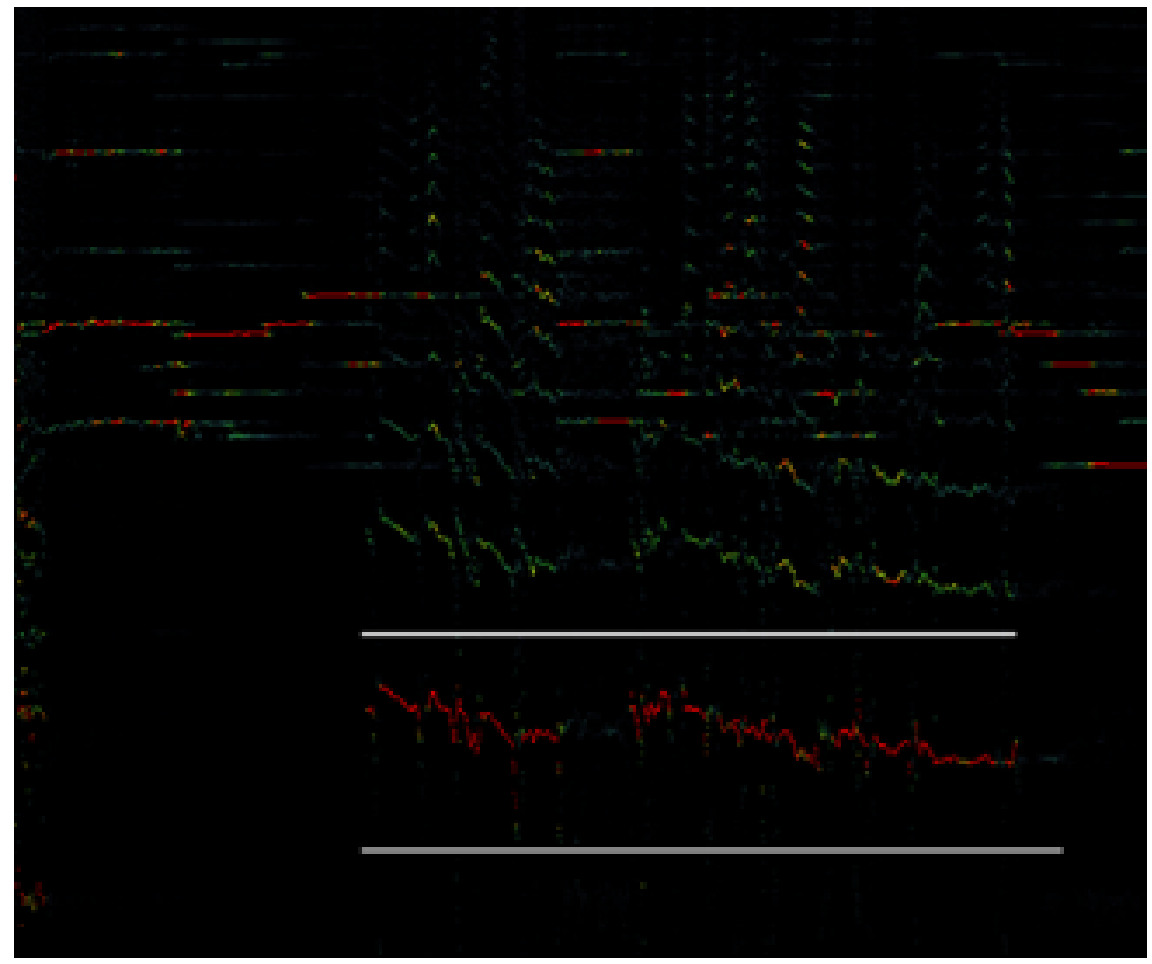

Es. 3. Pd. VII i, "Osanna, sanctus Deus sabaòth". Spettogramma (peak frequency).

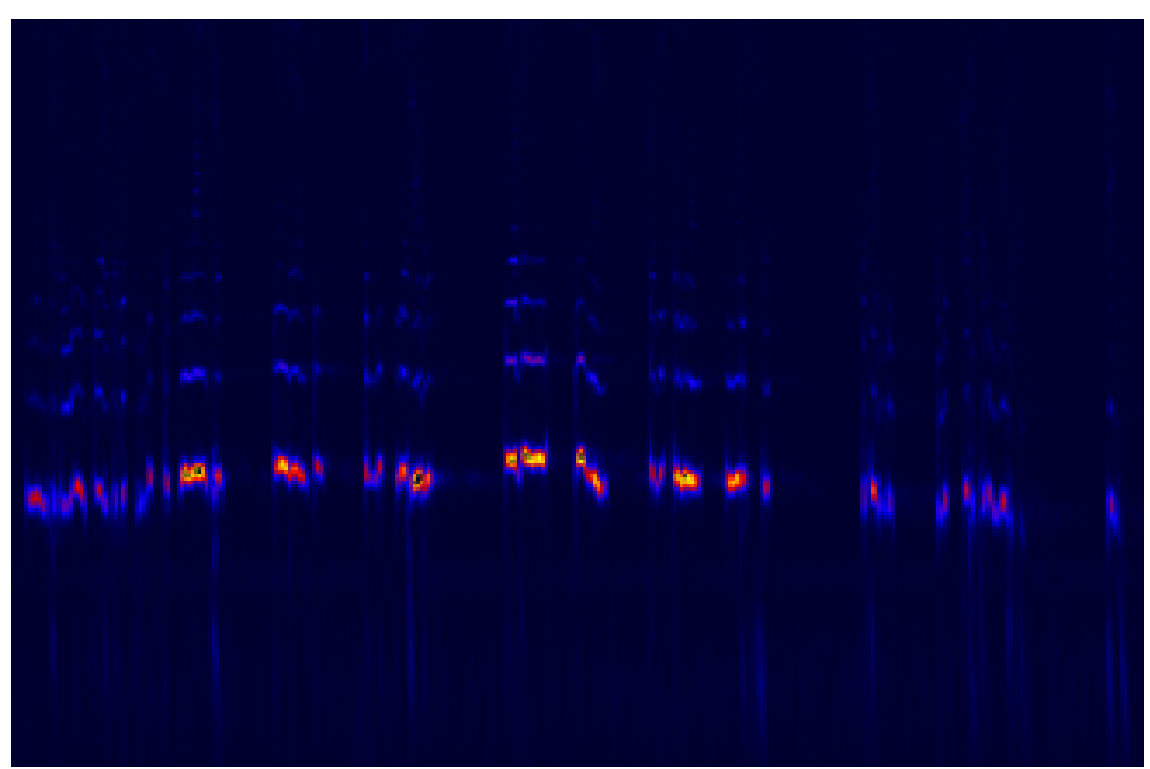

Es. 4. If. V I39-I42. Spettogramma (melodic range). 
E se nei frammenti della Divina Commedia Bene sembra trasmettere tutto il dolore per una ferita, coi sonetti restituisce pace a chi ascolta: letti come in un'atmosfera elettrica, essi vengono a sciogliere una tensione altrimenti insostenibile.

Nella ricerca di Bene la bocca si configura come punta del corpo; basti pensare al marcato e perentorio staccato col quale egli pronuncia la parola "bocca", nell'incipit del XXXIII canto dell'Inferno dedicato alla vicenda di Ugolino:

La bocca sollevò dal fiero pasto

Quel peccator, forbendola a'capelli

Del capo ch'elli avea di retro guasto. ${ }^{15}$

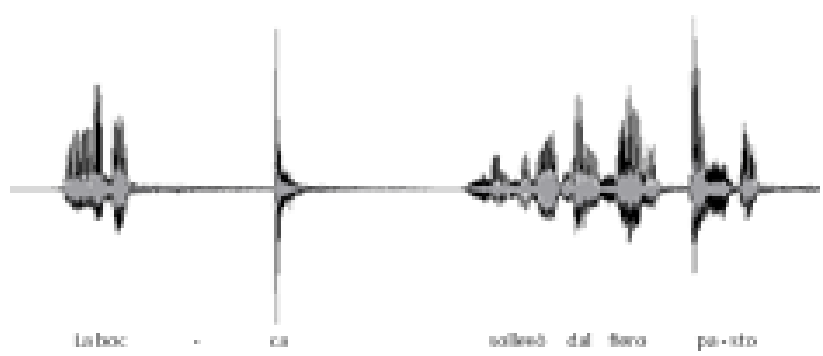

Es. 5. If. XXXIII I, "La bocca sollevò dal fiero pasto". Forma d'onda.

Bene sembra propendere per un "uso" minore di Dante, che ne evidenzi le deficienze, i balbettamenti, le improvvise esplosioni. Nel 1978 notava Deleuze, in Un manifesto di meno, l'insistenza beniana sulle protesi, le bende, le più $o$ meno metaforiche amputazioni: segni appunto di "un trattamento minore o di minorazione, per sprigionare dei divenire contro la Storia, delle vite contro la cultura, dei pensieri contro la dottrina, delle grazie o delle disgrazie contro il dogma" (Deleuze 2002: 9I). Nello specifico dantesco, l'insistenza di Bene a tratti fagocitante, "la continua ruminazione sillabica, lo snocciolarsi degli staccati" (Cortellessa 2004), fanno quasi pensare - suggerisce Andrea Cortellessa - all'etimo falso di Comedia: da comedere, divorare; o ancora, riprendendo il Mandel'štam di Conversazione su Dante, si potrebbe affermare che "il baricentro dell'attività discorsiva s'era spostato: verso le labbra, verso l'esterno della bocca. D'un tratto, la punta della lingua s'era trovata in una posizione di spicco" (Mandel'štam 2003: 123). La registrazione sonora della performance del 3I luglio 1981 ci restituisce una Divina Commedia diversa, che mostra a tratti la stupenda infantilità della fonetica italiana spesso vicina al farfugliare dei bambini, a tratti quel misto di roco e stridulo che si ode nelle voci degli

I5. La terzina è carica di tensione per la grande forza che si sprigiona d'attacco (la bocca avidamente attaccata alla testa del nemico) sino alla terribile immagine del cranio devastato dal morso. 
anziani, insieme a un certo suo congenito e raffinato dadaismo, attraverso un prospetto ritmico e timbrico sempre - e improvvisamente - cangiante. Come scritto da Piergiorgio Giacchè,

[Bene] somma l'invisibile e l'inaudito, dando suono musicale e senso vitale ai versi più alti del patrimonio letterario italiano e mondiale. Per la prima volta - quella volta - il miracolo ovvero lo spettacolo della "verticalità del verso" diventerà evidente perché liberato da un'iperbolica rampa di lancio, di cui poi non ci sarà più nessun bisogno ogni altra volta in cui la sua Lectura Dantis sarà riproposta pubblicamente (Giacchè in Bene 2005: 17I).

Bene stava resuscitando Dante come né prima né poi è mai riuscito ad altri (tra parentesi vale la pena - e pena in tutti i sensi - di ricordare i tanti fini dicitori spesso toscani che ancora cercano inutilmente di fare il verso di Dante e $a$ Carmelo). Bene stava resuscitando Dante - come gli è capitato e gli capiterà di fare con Majakovskij e Byron e Leopardi e Manzoni e Campana e D'Annunzio - ...ma soprattutto, consapevole o no, stava resuscitando la Piazza. Quella Piazza ferita a morte appena un anno prima, e da molti anni comunque assente dalla vita culturale e abbandonata dalla vita sociale (Giacchè in Bene 2005: 173).

E la registrazione sonora ci restituisce quel miracolo sotto forma d'opera d'arte, Dante e la Piazza in un solo attimo e atto: un'esperienza sonora e un'orazione civile tra le più alte che, a memoria d'Italia, a un attore sia mai riuscito di creare e inverare.

\section{Una breve apparizione: Salvatore Sciarrino}

In ultimo, avendo impropriamente, anche se necessariamente, portato Lectura Dantis sul piano della riflessione di carattere musicale, devo ricordare con una breve postilla che l'opera fu accompagnata anche da musiche composte per l'occasione da Salvatore Sciarrino. L'organico prevedeva strumenti registrati su nastro (in sovra-incisione) da un solo esecutore: flauti dolci (soprano, contralto, tenore e basso); flauto dolce sopranino; cromormo contralto e tenore; dolzaina e cornamusa.

Il compositore siciliano fu chiamato da Bene a restituire, attraverso il nastro magnetico, le risonanze degli strumenti sotto forma di nuvole sospese, "sfondi sonori continui ma cangianti su cui aleggiano brani medievali (a loro volta intermezzi musicali per le 'letture' beniane)" (Angius 2002: 50). ${ }^{16}$ Sciarrino realizzò docici brevi minuti di musica che, in forma di frammenti, Bene antepose a ciascuna lettura, quasi a dar vita a una sorta di "melologo" a metà, in cui la musica, dal vago sapore arcaico, ha la funzione di introdurre e sot-

I6. Interessante notare come Angius, pur senza far riferimento alla tipologia di perfomance prodotta da Bene in Lectura Dantis, sottolinei la parola "letture", a rimarcare la non riducibilità a mera "lettura" dello spettacolo prodotto in quella occasione. 
tolineare il passaggio da una lettura all'altra. Si trattò del primo incontro del compositore siciliano con i versi della Commedia: un incontro tutto al servizio di Bene e del suo cantar recitando, al quale Sciarrino partecipò in punta di piedi, quasi fosse un nocchiero semplicemente chiamato a condurre l'ascoltatore da un canto all'altro, aprendo attraverso la musica uno spazio alla parola di Dante e alla voce di Bene.

A partire da Lectura Dantis, e per oltre un decennio, il poeta rappresentò per Sciarrino un punto fermo e stabile nella storia della cultura, una figura la cui opera si colloca in modo granitico nell'evoluzione delle forme artistiche. A dimostrazione del fascino esercitato da Dante sul compositore, basti un dato: nessuno lavoro su Dante, dei diversi prodotti da Sciarrino, è valutabile come un'esperienza isolata all'interno della carriera dell'artista siciliano, né tanto meno come un bozzetto che ha preso vita in forma precaria, frutto di un interesse passeggero e svagante a intervallare brani più rilevanti. Sciarrino fu intimamente coinvolto da Dante, dalla forma delle cantiche, da quello che esse raccontano, dall'ambiente sonoro che descrivono, dai suggerimenti infiniti che concedono.

Lectura Dantis fu il punto d'inizio di un percorso di scoperta e conoscenza: ancora il nastro magnetico fu protagonista nello stesso anno di La voce dell'Inferno, opera nata come originale radiofonico e poi diventata autonoma. ${ }^{17} \mathrm{Nel}$ prologo lo spettatore si trova come in un aeroporto, pronto a imbarcarsi, ma il rombo assordante del motore di un velivolo si trasfigura in musica, proiettando così l'ascolto in un luogo astratto; in questo luogo, dopo un suono di passi, quasi si fosse giunti sull'orlo di una voragine, si è come investiti dalla voce dell'Inferno: manipolate elettronicamente, appaiono le figure, gli ambienti e le situazioni della prima cantica.

Nel 1987 Sciarrino compose e incise per la Rai Sui Poemi concentrici I, II, III per solisti e orchestra. ${ }^{18}$ L'anno successivo furono mixati su nastro magnetico col titolo Musiche per la Divina Commedia di Dante Alighieri, in modo da sostenere l'intera recitazione dei testi per un totale di is ore complessive. ${ }^{19}$ Nel 1993 l'ennesima fatica su soggetto dantesco culmina nelle Musiche per il Paradiso di Dante (Alfabeto oscuro, L'invenzione della trasparenza, Postille), per orchestra con solisti. ${ }^{20}$

17. Salvatore Sciarrino, La voce dell'Inferno, per nastro magnetico, I98I; testo di Dante Alighieri. "XXIII Premio Italia", 2 settembre 198I (elaborazione elettronica del nastro realizzata il i9 giugno 198I presso lo Studio di Fonologia della Rai di Milano, tecnico del suono Marino Zuccheri); cfr. Broglia 2010 e Carratelli 2012.

I8. Salvatore Sciarrino, Sui Poemi concentrici I, II, III, per solisti e orchestra (Città di CastelloTorino, 1987); cfr. Angius 2002: 50-56.

19. Salvatore Sciarrino, Musiche per La Divina Commedia di Dante Alighieri, missaggio su nastro per le cento puntate televisive (Rai, Raitre Dipartimento Scuola Educazione). Letture di Giorgio Albertazzi (Inferno), Giancarlo Sbragia (Purgatorio), Enrico Maria Salerno (Paradiso). 20. Salvatore Sciarrino, Musiche per il Paradiso di Dante. Alfabeto oscuro, Linvenzione della tra- 
Viene naturale domandarsi il perché di tante occasioni dantesche. Come traspare dall'ascolto delle opere, a Sciarrino preme - per certi versi proprio come a Bene - l'urgenza di misurarsi con queste architetture poetiche dal grande impatto formale, ma anche di rifuggire dalla banalizzazione del puro "commento" sonoro: "a me pare che quando si affronta la globalità della Commedia, un vincolo progettuale si impone al compositore. Una musica che seguisse questi poemi parola per parola non avrebbe senso estetico: essi non si lasciano trattare come un qualsiasi testo. Bisogna semmai creare con la musica uno spazio per la parola di Dante". ${ }^{2 \mathrm{I}}$

Tanto l'attore Bene, quanto il compositore Sciarrino, ponendosi per scelta "fuori dell'opera", non si comportano certo come un cantante da palcoscenico né tantomeno come un suonatore d'orchestra: non compiono gesti che accompagnino atleticamente l'attraversamento dantesco, né peggio che illustrino personaggi o incoraggino significati. Essi tentano di risolvere il problema della propria umana inadeguatezza di fronte all'oltremondano cammino verso il $\mathrm{Pa}$ radiso, azzerando o, in altri casi, protestando la propria irriducibile presenza.

\section{BiBLIOGRAFIA}

Angius, M., 2002, "Sui poemi concentrici. Da Sciarrino a Dante (con un'apparizione di Carmelo Bene)", Hortus Musicus, III/12, pp. 50-56.

Bene, C., 198I, Otello, o la deficienza della donna, Milano, Feltrinelli.

—, 2005, Sono apparso alla Madonna, Milano, Bompiani.

— , e G. Dotto, I998, Vita di Carmelo Bene, Milano, Bompiani.

Benjamin, W., 20I4, L'opera d'arte nell'epoca della sua riproducibilità tecnica, Torino, Einaudi.

Broglia, S., 20Io, "'Urlar li fa la pioggia come cani'. Ambiente sonoro, voce ed elettronica nell' Inferno di Sciarrino", Doctor Virtualis, IO, pp. 99-I23.

Caporaletti, V., 2000, La definizione dello swing. I fondamenti estetici del jazz e delle musiche audiotattili, Teramo, Ideasuoni.

—, 2005, I processi improvvisativi nella musica. Un approccio globale, Lucca, LIM.

— , 20ıo, "Esperienza audiotattile e molteplicità della musica", B@belonlinelprint, 8 (Musica e Bildung), pp. 5I-63.

Carratelli, C., 20I2, "La voce dell'Inferno di Edoardo Torricella e Salvatore Sciarrino", in Il prix Italia e la sperimentazione radiofonica: l'immaginazione in ascolto, a cura di A. I. De Benedictis e M. M. Novati, Roma, Die Schachtel-Rai Trade, pp. 97-II3.

Cortellessa, A., 2004, "Un muezzin per Dante", Alias de Il Manifesto, io luglio.

Costa, G. (a cura di), 2003, A CB. A Carmelo Bene, Roma, Editoria\&Spettacolo.

De Kerckhove, D., I99I, Technology, Mind and Business, Utrecht, Bosch \& Keuning. Deleuze, G., 2002, "Un manifesto di meno", in C. Bene e G. Deleuze, Sovrapposizioni,

sparenza, Postille (Città di Castello, 1993), drammaturgia di Giovanni Giudici, protagonista Sandro Lombardi, I Magazzini, regia di Federico Tiezzi, Ravenna Festival 1993.

2I. Salvatore Sciarrino, Sui Poemi concentrici, note di sala dell'autore. 
Macerata, Quodlibet, pp. 87-II6.

Fofi, G., e P. Giacchè (a cura di), 1995, Per Carmelo Bene, Milano, Linea d'ombra.

Giacchè, P., 2007, Antropologia di una macchina attoriale, Milano, Bompiani.

Grande, M., I982, "L'uso della strumentazione elettronica nell'ultimo Bene", in Il suono del teatro, a cura di R. Tomasino, Palermo, Acquario, pp. 45-73.

Guarnieri, G., I993, Il teatro in contropiede. Cronache e scritti teatrali 1974-1981, a cura di S. Chinzani, Roma, Bulzoni.

Maenza, R., 20I2, "Un 'bene’ grande per Bologna. Carmelo Bene sopra le Due Torri”, in 05I. 20I2-I970: Bologna, identità e memoria, a cura di G. Santarelli, Bologna, Bononia University Press, pp. 220-227.

Mandel'štam, O., 2003, Sulla poesia, Milano, Bompiani.

McLuhan, M., 1962, Gutenberg Galaxy: The Making of the Typographic Man, Toronto, University of Toronto Press.

McLuhan, M., 1964, Understanding Media: The Extensions of Man, New York, McGraw-Hill.

McLuhan, M., e E. McLuhan, 1988, The Laws of Media: The New Science, Toronto, University of Toronto Press.

Meletti, J., 20Io, "Strage. Un anno dopo il 2 agosto l'orazione civile di Carmelo Bene”, La Repubblica, II ottobre. 